Techniques \& Culture

Revue semestrielle d'anthropologie des techniques

62 | 2014

Le corps instrument

\title{
Apprendre à faire le clown
}

The art of doing the clown

\section{Philippe Hert}

\section{OpenEdition}

\section{Journals}

Édition électronique

URL : https://journals.openedition.org/tc/8285

DOI : $10.4000 /$ tc. 8285

ISSN : 1952-420X

Éditeur

Éditions de l'EHESS

Édition imprimée

Date de publication : 1 décembre 2014

Pagination : $30-47$

ISBN : 978-2-7351-2346-9

ISSN : 0248-6016

Référence électronique

Philippe Hert, « Apprendre à faire le clown », Techniques \& Culture [En ligne], 62 | 2014, mis en ligne le 01 décembre 2017, consulté le 30 septembre 2022. URL : http://journals.openedition.org/tc/8285 ;

DOI : https://doi.org/10.4000/tc.8285 


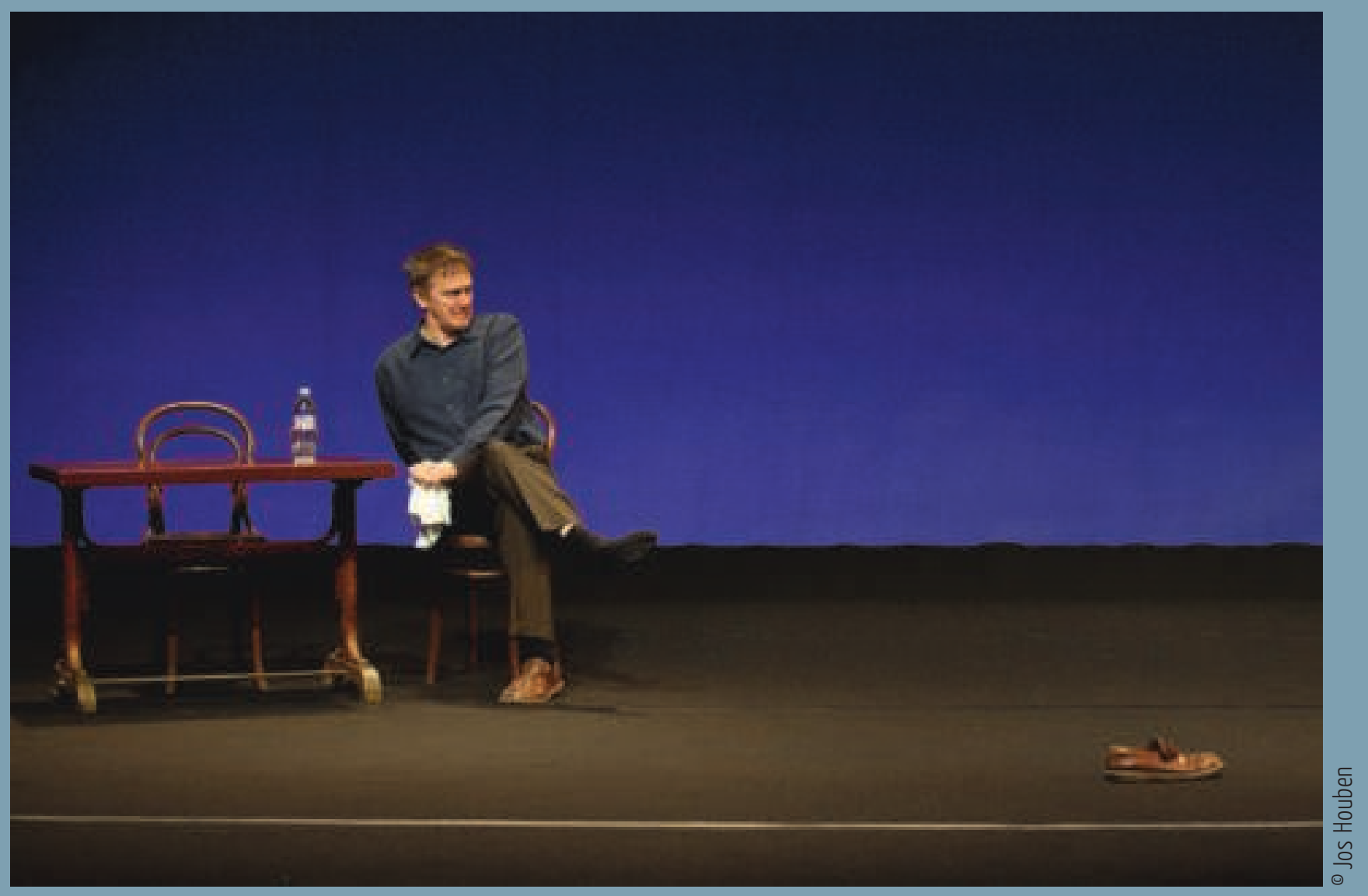




\section{APPRENDRE À FAIRE LE CLOWN}

La pratique du clown renvoie souvent à une figure de personnage portant un nez rouge, maquillé, avec un costume bariolé. Les formes du clown contemporain, également appelé «nouveau clown» (Cézard 2014) ne correspondent pas à ces représentations collectives populaires et stéréotypées. Le clown contemporain que j'explore ici peut se présenter sans nez, ni maquillage, avec un costume de scène qui ne renvoie pas forcément au monde du cirque. Ce n'est pas l'habit qui fait le clown. Au-delà de ces formes sociales attendues, l'intérêt que je porte à la pratique du clown contemporain a été motivé par une parole de clown rencontré lors d'un stage: «ce sont les clowns qui sont drôles, pas ce qu'ils font ${ }^{1} »$. Cette parole intrigante m'a mené vers plusieurs autres stages et formations de clown, pour explorer cette pratique, à la fois chargée de conventions sociales et, pour sa forme contemporaine, très libre dans son mode d'improvisation. La question qui m'intéressait alors portait sur ce qui motive les participants d'un stage à venir apprendre à faire le clown ou plus justement «apprendre le clown». Mais au lieu de mener une enquête qualitative ou quantitative sur ces motivations, il m’a semblé plus intéressant de commencer l'analyse par la pratique elle-même, en l'expérimentant afin de sentir les «prises» disponibles lorsque l'improvisateur est engagé dans un jeu clownesque. Cette notion de prise, proposée par Bessy et Chateauraynaud $(1995,2010)$ s'inscrit dans une analyse pragmatique de l'action située. Elle me servira ici pour indiquer les moyens dont dispose le clown pour exprimer son jeu dans sa relation avec le public. Je la convoque car elle possède le grand avantage d'être symétrique et réflexive, comme le souligne Hennion (2009) : elle renvoie à la fois à l'action d'avoir prise et à l'état d'être pris (par une situation, des contraintes, un environnement), donc une certaine passivité dans l'action elle-même, 
ce qui est important, pour saisir le travail du clown. D'ailleurs le mot clown, tout comme le mot performance, sont également symétriques: ils désignent à la fois le processus et le résultat de l'action. En observant quelles prises offre la pratique du clown, j'ai tenté de comprendre la motivation à devenir clown. Cette analyse est le fruit d'une pratique en clown et des retours des professionnels qui m'ont encadré, complété ensuite par des entretiens qualitatifs avec des stagiaires et des clowns-enseignants. Cette exploration peut ainsi se rapprocher de l'analyse de l'attachement que propose Hennion: «technique collective pour se rendre sensible aux choses, à son corps, à soi-même, aux situations et aux moments, tout en contrôlant le caractère partagé ou discutable de ces effets avec les autres, c'est mettre la réflexivité du côté des amateurs - et non pas seulement des sociologues soucieux de ne pas biaiser leurs analyses» (Hennion 2009: 56).

Plutôt que d'essayer de définir une spécificité du jeu du clown par rapport à d'autres formes, dans le monde du spectacle vivant très mouvant, qui croise les approches plutôt que de les cloisonner, je préfère me concentrer sur le jeu corporel du clown, que l'on peut retrouver dans le jeu d'acteur ou de comique. De plus l'apprentissage du clown peut faire partie de la formation d'acteur. L'école internationale de théâtre Jacques Lecoq fait figure de précurseur, en intégrant le jeu du clown dans le jeu de l'acteur. Je m'intéresse donc à l'être clown en essayant de définir une catégorie heuristique: j’adopte à la fois un point de vue intérieur et extérieur, qui dépasse les représentations sociales du clown, pour me concentrer sur les techniques du corps qu'il développe. Ainsi, pour décrire des manières d'apprendre le clown, j'articulerai mon propos autour de techniques du corps au sens de Mauss, dont je reprendrai plus loin l'analyse qu'en fait Sigaut, en tant qu'actes corporels traditionnels et efficaces, autrement dit, qui font l'objet de transmissions - objet des stages - et de performances socialement attendues et validées. Avant d'aborder ces techniques du corps du clown, explicitons quelques points pour préciser cette parole citée précédemment. Si les actes du clown ne sont qu'un prétexte, qu'est-ce qui est drôle chez le clown? Quelles sont ses prises?

\section{Ce qui fait le clown}

\section{Le personnage et le prétexte}

Le jeu du clown est construit sur un prétexte: ce qui constitue le canevas de la scène à jouer, basé sur une mise en situation, des objets, un enjeu. Le jeu du clown est incarné par un acteur. Cependant, celui-ci jouit d'une autonomie dans le sens où l'acteur ne doit pas intervenir dans ce que vit le clown. Il faut distinguer trois places dans le jeu du clown. Lacteur travaille pour que le clown se libère, et l'auteur en fait une matière pour écrire un spectacle (je laisserai ici de côté les questions relatives à l'auteur et à l'écriture d'un spectacle de clown). Le clown est plus qu'un personnage de théâtre, car il fait partie de l'acteur. L'acteur doit accepter d'abandonner toute volonté de contrôle, de maîtrise sur le jeu du clown ${ }^{2}$. Mais loin de tomber dans l'injonction paradoxale du «sois spontané», cet abandon suppose un travail sur le corps. C'est pourquoi parmi les apprentis clowns la question est souvent posée: «as-tu trouvé ton clown? »Cela indique que chacun 
devrait trouver «son » clown. Mais cette idée induit une confusion importante: le clown pourrait être considéré comme un personnage inventé que chaque apprenti devrait construire et incarner. Or, celui-ci n'est pas un personnage, mais le reflet de sa propre vulnérabilité.

«Le clown n'existe pas en dehors de l'acteur qui le joue. Nous sommes tous des clowns, nous nous croyons tous beaux, intelligents et forts, alors que nous avons chacun nos faiblesses, notre dérisoire, qui, en s'exprimant, font rire» (Lecoq 1997: 153).

On devient clown en arrêtant de jouer l'idée que l'on a d'un personnage de clown. Il s'agit d'apprendre à laisser exister sur scène ses propres faiblesses. Si le clown n'échappe pas vraiment au paradoxe de l'acteur, ce dernier laisse néanmoins paraître ses propres sensations ou émotions dans le jeu, dans sa relation avec le public, avec son ou ses partenaires de jeu, avec des objets. De ce fait, le clown est un expérimentateur permanent; l'improvisation est pour lui une manière d'explorer des formes de rapport au monde, en relation avec un public. Il fait un travail de performance sur sa présence clownesque. On comprend mieux alors pourquoi il n'existe pas de conservatoire de clown et pourquoi chaque clown est unique. D'après Michel Dallaire ${ }^{3}$, le clown exprime spontanément et ne se construit pas. Cependant, le clown a une maîtrise de sa présence scénique, il ne fait pas juste n'importe quoi. Il semble donc qu'un paradoxe existe entre la présence unique et spontanée du clown

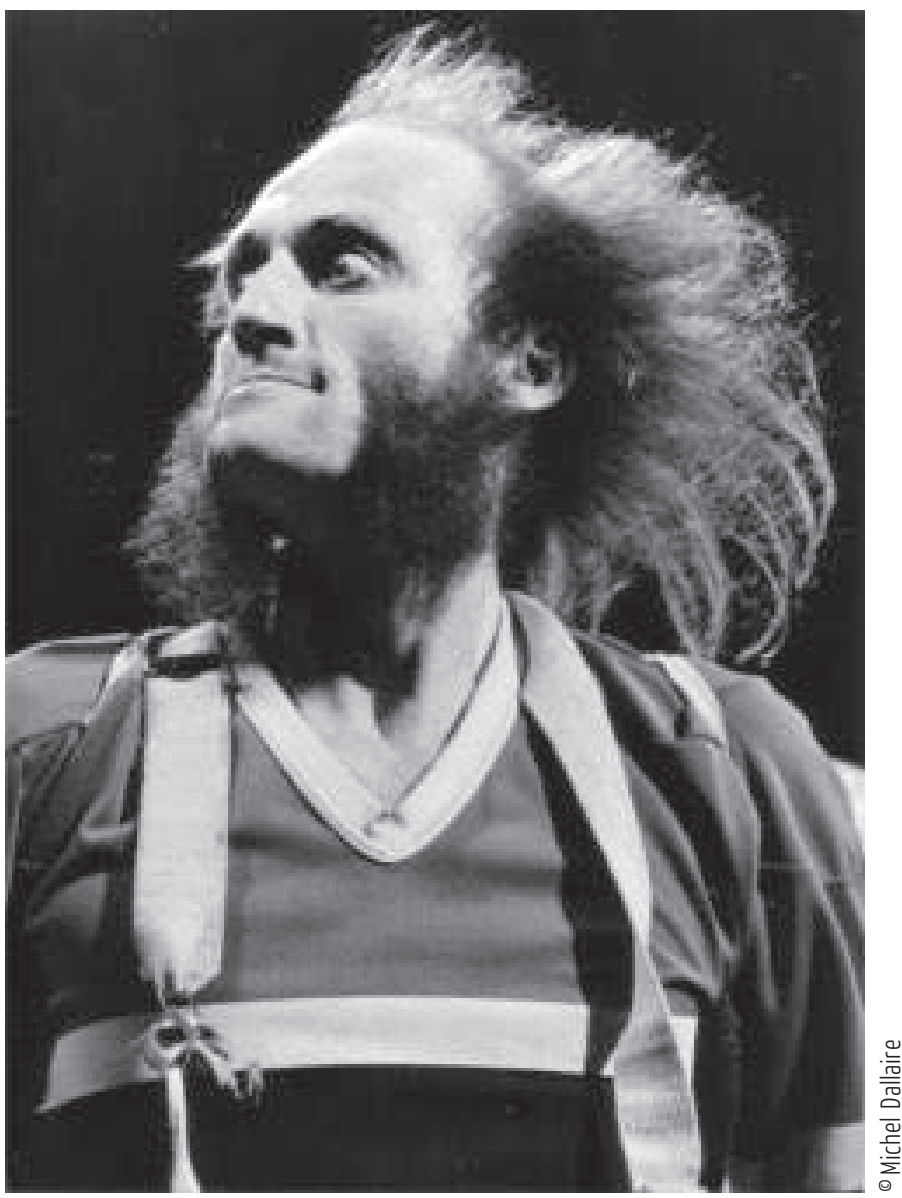
et la construction d'un jeu scénique, dans l'optique d'un spectacle. Ce paradoxe constitue une piste intéressante pour comprendre le travail du clown. Celui-ci ne se résume pas au paradoxe de l'acteur qui consiste à «jouer vrai» sur scène, car pour le clown le jeu sur scène est vrai, au sens où il témoigne de ses propres émotions et sensations. Dans le jeu à plusieurs clowns, ce que ressent et communique un clown «donne du jeu » à son ou ses partenaires, permettant à un jeu d'improvisation «en famille» de s'exprimer.

\section{Se donner un cadre pour être spontané}

Comment s'apprend cette présence spontanée? La liberté laissée au clown par l'acteur fait l'objet d'apprentissages dans les stages et les formations professionnelles. J'indique ici des éléments d'un parcours d'apprentissage personnel, sans chercher à être exhaustif. S'il s'agit de moyens pour stimuler le jeu en improvisation qui reste personnel et intime, les manières de construire un personnage identifiable à un clown sont cependant collectives et socialement partageables. On peut distinguer les formations professionnelles de clown des stages amateurs, par rapport à l'attente vis-à-vis des stagiaires, sur le plan de leur maîtrise corporelle et de leur présence scénique. Toutefois, les personnes suivant 
une formation professionnelle de clown viennent souvent d'un autre domaine artistique (théâtre, cirque, danse, etc.) et cherchent à en connaître les spécificités. À l'exception des stages de découverte ou d'initiation, le travail d'apprentissage amateur ou professionnel se rejoint du point de vue de la mise en place d'un cadre qui favorise l'expression du clown. Les stages comportent en général un travail de préparation physique, individuel et collectif, des jeux relationnels qui permettent des interactions avec les autres apprenants, pour une mise en confiance, un travail d'improvisation collectif, puis en duo, et enfin en solo, sur le plateau (cet ordre est bien sûr optionnel). Les improvisations sont les temps forts où chacun peut expérimenter face à un public. Elles partent d'un prétexte, généralement donné par l'enseignant (une salle d'attente chez le dentiste, retrouver sa chambre d'enfance, etc.). Au besoin, le formateur peut donner des indications à l'acteur pendant son improvisation, lui poser des questions ou lui suggérer des pistes qui vont l'aider dans l'expressivité de son clown, et consacrer un temps de retour collectif après les scènes improvisées. Il s'agit d'occasions pour expérimenter des comportements peu verbalisés qui peuvent toucher à l'intime, et être vécus comme une «mise à nu» de la personne. Ainsi, certains stages de clown sont davantage assimilés à du développement personnel qu'à la recherche d'une performance artistique. Je n'aborderai pas cet aspect du clown ici, pour pouvoir me concentrer sur les apprentissages corporels.

Lors de ces temps de parole collective, l'impression de transparence pour le public des sentiments que traverse le clown sur scène est souvent formulé: le public perçoit finement des micro-expressions d'états émotionnels, à un degré que le clown débutant n'imagine pas lorsqu'il est sur scène. Car bien sûr être clown sur scène produit des émotions: être face à un public, même bienveillant, sans filet, constitue une prise de risque pour son ego, son image sociale. Il s'agit d'apprendre à percevoir ces émotions fugaces du clown et de ne pas les laisser filer. Lacteur clown débutant a souvent tendance à proposer beaucoup de pistes au public, à le noyer de propositions pour ne pas montrer sa peur. Il est sous la pression de la scène, ce qui l'empêche de laisser son clown déployer une prise et de le laisser vivre avec. Un des premiers apprentissages consiste justement à resserrer son jeu, à en faire moins - sauf si cela est justement une proposition clownesque d'en faire trop.

\section{La place du public}

Quel rôle joue ce public dans le jeu du clown? Selon une comédienne formée au clown: «le clown est un jeu à trois, il y a toujours ton clown, le prétexte et le public. Ensuite il peut y avoir un partenaire de jeu qui peut s'ajouter. La même scène n'est jamais jouée pareille avec un public différent [...] il y a toujours un aller-retour avec le public ${ }^{4} »$. Ce témoignage rend compte d'une entrée dans le clown à partir de l'expérience du solo sur scène comme étant en réalité un duo avec le public. Le public joue le rôle de validateur pour le clown. Ses réactions, ses rires, son attention plus ou moins forte, sont autant de signes qui encouragent le jeu du clown dans un sens ou un autre, ce qui rend la performance du clown singulière.

L'épreuve de la scène est constitutive du clown, quelle que soit la scène par ailleurs. Le clown a besoin d'une altérité qui le confirme dans sa place. C'est la première prise pour apprendre le clown: si le public (les autres stagiaires par exemple) rit, on est sur la bonne voie. Le test du public peut être à la base de l'écriture d'un spectacle. Ce rire est moteur pour le clown qui doit apprendre à garder l'état de corps dans lequel il se 


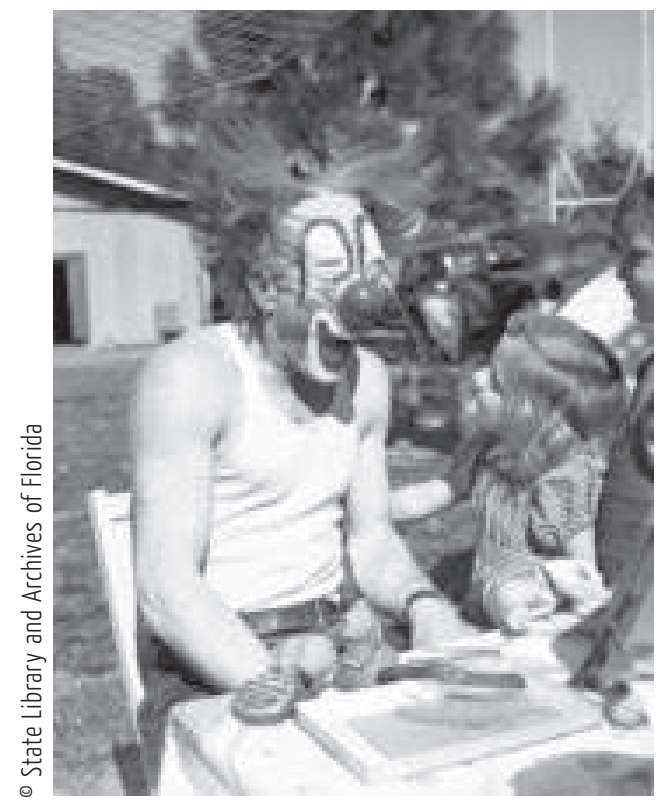

Lou Jacobs, le clown du Ringling Circus avec Carla Wallenda, Sarasota, Florida, 1941

Le jeu du clown repose sur un rapport permanent avec un public qu'il s’agit d'apprivoiser.

trouve au moment où il fait rire l'auditoire. Le clown a un regard direct vers le public, il l'interpelle et éventuellement le fait participer. Ce que cherche le clown par là, c'est le partage de ce qu'il vit dans son corps au moment présent, sur scène; ce qui lui permet éventuellement d'improviser avec le public. Le clown qui a trouvé «un truc qui marche» avec ce public particulier doit apprendre à maintenir cet état. La conscience du clown porte sur cet état de corps, et pas (ou peu) sur ce qu'il va dire, à la différence du comique de spectacle ou de théâtre. C'est ici que la notion de prise est éclairante: la scène, improvisée ou construite dans une écriture scénique, offre des prises pour que le clown puisse s'exprimer, et pour que, symétriquement, l'acteur se laisse prendre au jeu de son clown. En termes de prises, il s'agit en fait souvent d'arriver à un certain lâcher prise. La contradiction n'est qu'apparente, car pour se retrouver en position de donner prise au clown dans une situation précise, en fonction des réactions du public, il s'agit de se laisser prendre à son jeu. On retrouve bien la symétrie du terme. Le lâcher prise concerne souvent le fait de se défaire de l'idée que l'acteur se représente de son jeu de clown, pour pouvoir laisser exister la situation, sans faire exécuter une chose précise à «son» clown, imaginant une scène à l'avance en se disant qu'elle sera drôle. Dans ce dernier cas, en général c'est l'échec assuré, et par bonheur, le public n'est ni dupe ni indulgent, au plus grand profit de l'apprenti acteur! C'est également en ce sens que la recherche de «son» clown est probablement vaine.

Le clown a par conséquent besoin d'un public. Même si le spectacle est déjà écrit, ce temps d'interaction est indispensable pour que le clown puisse prendre la mesure de son geste et son attitude, plus ou moins en décalage, plus ou moins ridicule, plus ou moins distanciée. Il peut ainsi montrer ou dire au public ce qu'il est en train de vivre en s'adressant à lui et en le regardant. Lorsque les stagiaires observent un des leurs travailler sur le plateau, ils sont également en train de travailler le clown; en prenant le rôle de public, ils apprennent à voir l'être clown qui apparaît devant eux à travers ce qui les fait rire. Le rire est ici un mode de communication entre le public et le clown: le public 
exprime ainsi que ce qu'il voit lui plaît, et plus profondément qu'il se reconnaît dans les attitudes qu'il capte, et qu'elles sont donc justes. Le rire est à la fois une réaction à ce qui est montré, mais également une façon de dire au clown qu'il peut continuer. Si le faire-rire n'est pas la seule finalité du clown, elle reste néanmoins importante: sans rire, le clown ne peut plus avoir de jeu et ne sait plus s'il est juste. D'ailleurs, en formation, il est souvent indiqué au clown de ne pas «sucer son public », c'est-à-dire de ne pas le prendre en otage en quelque sorte en lui demandant implicitement de valider chacune de ses propositions. Il n'est plus dans le jeu expressif du clown à ce moment-là, ni dans le jeu d'acteur, il joue alors uniquement en réaction. De son côté, le public vient voir un clown pour rire, c'est certain. Cependant, il ne cherche probablement pas seulement une distraction. La position de public adoptée par les stagiaires en témoigne, même s'il s'agit en l'occurrence d'un public particulier. Ce qui nous touche lorsque nous voyons un clown, est qu'il nous renvoie à notre commune humanité; il nous rappelle des aspects de nous qu'il nous offre en partage. Dès lors l'identification du public au clown se fait dans un jeu subtil et conjoint, entre distanciation, par son ridicule, et empathie, par la proximité de ce qu'il montre avec nos propres vies et sa manière de nous le montrer. Inversement, le rapport du clown au public est un autre jeu subtil de proposition de jeu et d'écoute de sa validation par le public. Si le clown cherche à faire rire, il n'est pas juste une marionnette comique et ridicule. Cette présentation du jeu du clown est certainement très incomplète, car elle focalise sur un art de faire rire, alors qu'il existe également une dimension poétique dans le clown que je n'aborderai pas ici. À ce propos, Catherine Germain dit de son clown Arletti (Cervantès \& Germain 2009: 48) : «le clown n'est pas un acteur. Le clown est un poète, et même s'il est accompagné, entouré et conseillé pendant la création de ses spectacles, au bout du compte, son acte est absolument personnel et authentique [...]. Le clown ne dit pas un poème, il ne fait pas un poème, il "est" un poème.»

\section{L’apprentissage corporel pour être unique sur scène}

Si le clown est poème, il semble vain de lister ce que seraient des «techniques», au sens trivial de méthodes, pour faire le clown. Je cherche au contraire à préciser, à travers un parcours personnel et subjectif, comment se construit un savoir être clown par un travail du corps. Plus précisément, des techniques du corps peuvent-elles être décrites pour parvenir à un jeu de clown? Autrement dit, quels sont les actes efficaces appris en formation qui permettent de faire exister un être clown? Nous avons vu l'importance pour le clown de lâcher prise par rapport à une idée qu'il se figure de ce que fait un clown. Le travail du clown porte donc moins sur une représentation de soi que sur l'exploration d'un état de corps. Pour qualifier cet état de corps, certains clowns empruntent au Body Mind Centering $(\mathrm{BMC})^{5}$, l'exploration sensitive de leur propre corps. Ils parviennent ainsi à caractériser des états de corps sur de nombreux plans: émotionnel, sensitif, musculaire, nerveux, osseux, réflexe, organique (au sens de la perception interne des organes comme le cour, les intestins, les poumons, la peau), etc.

Nous allons voir des techniques du corps mobilisées en clown pour explorer ces états de corps. Les exercices d'improvisations déjà évoqués, servent à travailler l'expressivité du 


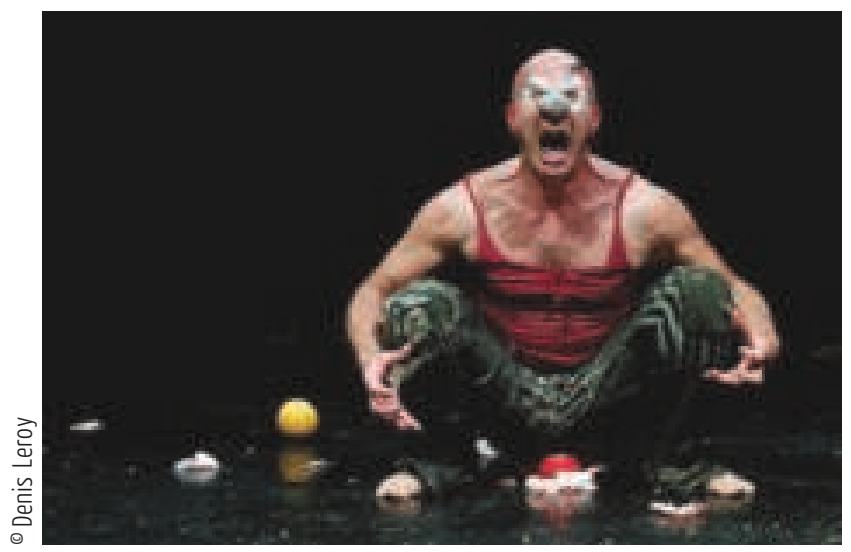

\section{Ludor Citrik}

Un travail sur l'expressivité corporelle

du clown

clown pour convoquer son corps et non une image qu'il s'en fait, ou qu'il se fait du clown. Ceux-ci mettent en œuvre, à côté de la temporalité, du rythme, de la tension, des silences, des décrochages, l'état de corps du clown sur lequel nous portons plus précisément notre intérêt. Les formations de clown partent du jeu spontané de chacun, ou du jeu en interaction entre deux clowns. Ce n'est qu'ensuite qu'intervient la construction dramatique: rythme, durée, attaques, relances, écoute, tensions, états, étapes de jeu. Ainsi, dans un premier temps, il s'agit de vivre des situations sans se préoccuper du cadre de jeu. Le clown apparaît lorsqu'il partage avec le public ces situations qu'il vit. Une acrobate se formant au clown explique ce qui l'a attirée: «On ne peut pas tricher dans le clown, on ne joue pas à l'être, on l'est, dans l'instant présent... C'est toujours une joie pour moi quand j'y arrive et je retrouve mon personnage de clown ${ }^{6}$.» Lapprentissage de cette présence dans l'instant, repose évidemment sur une présence physique, un état de corps: le clown se montre dans ses attitudes, ses gestes, les sons qu'il produit, qui expriment ses états physiques, ses sensations, ses sentiments, ce qu'il vit face au public. La spontanéité du clown, c'est la parole donnée au corps. Cette parole est offerte au public: voici ce que je vis, maintenant, devant vous. Bien sûr cet état de corps est prévu à l'avance, l'acteur sait qu'il doit arriver à un certain état à un moment précis de son jeu sur scène, mais il reste que le clown travaille d'abord son expressivité corporelle. Ainsi, Ludor Citrik dans Je ne suis pas un numéro, joue du décalage entre son physique massif dans une robe légère, et un corps crispé formulant sa demande tantôt suppliante, tantôt énervée adressée au public: «je veux un câlin ${ }^{7}$ ».

\section{Le saut dans le vide}

Une première technique du corps pour travailler, produire et rendre visible efficacement des états de corps, est celle du «saut dans le vide»: elle consiste à agir sans savoir à l'avance quoi faire. Pour cela, il convient de poser des contraintes de jeu. La première consiste à devoir arriver sur le plateau, de sortir du fameux rideau de scène, sans préméditation, et de prendre ce qui vient à lui : ses réactions face au public ou face à des objets ou d'autres clowns, ainsi que les réactions du public. L'apprenti clown n'a pas le droit d'être passif, il doit absolument faire des propositions. Une manière de corser le jeu est de poser l'injonction de faire rire le public en un temps limité. Un enseignant dit à ce propos que «le clown saute dans la piscine sans savoir s'il y a de l'eau dedans ${ }^{8}$ ». 
Injonction difficile pour le débutant, car la tentation est grande pour l'acteur de vouloir faire quelque chose qui correspond à sa propre idée du clown. Cette manière de se lancer dans l'action sans réfléchir est efficace parce qu'elle permet d'enlever ou de diminuer la volonté de l'acteur d'avoir prise sur son clown. Une seconde contrainte consiste à fixer un objectif impossible à l'acteur, comme lui demander de venir expliquer au public qu'il est le roi des techniques de relaxation, sans qu'il sache par ailleurs ce que sont ces techniques. C'est alors au clown de prendre le relais, en montrant que tout va bien se passer, au moment où l'acteur défaille dans sa prétention. Le clown peut à loisir défaire ce que fait l'acteur, pour le plus grand plaisir du public et pour sa plus grande jubilation. Lorsque le clown est plus à l'aise, une troisième contrainte consiste à mettre de l'enjeu dans l'improvisation, et de jouer le conflit: par exemple l'enjeu pour l'acteur de garder sa dignité dans une situation ridicule. Toutes les défaillances de l'acteur sont autant de prises pour le clown afin qu'il s'exprime. Cet écart est exploité dans le jeu clownesque pour montrer des situations de conflit interne; il peut donner lieu à l'expression de multiples sensations et émotions dont va s'emparer le clown. Ces sensations et émotions sont clairement recherchées pour qu'un état de corps du clown intéressant apparaisse: tremblements, excitation, crispation, hésitation, balancements, etc., éventuellement accompagnés d'émotions de jubilation, de colère, de panique, de peur, de découragement, etc. En d'autres termes, cela signifie pour l'acteur de sortir de sa «zone de confort » de jeu, de se mettre en situation de risque afin de ressentir ce risque et donner alors du jeu au clown. Cette prise de risque reste toutefois liée au plaisir de jeu éprouvé par l'acteur. Tous ces éléments permettent un jeu organique et expressif du clown. Avec une telle approche corporelle du clown, il n'y a plus lieu de parler de «son» clown, mais simplement d'une personne qui s'incarne dans un clown.

Un tel apprentissage du clown explore un mode d'existence physique, des modes d'expression; il implique un plaisir, une jubilation dans le jeu. Si l'acteur peut douter, notamment vis-à-vis d'un jugement négatif du public, le clown n’a pas de doute quant à ce qu'il vit sur l'instant. Le doute ferait immédiatement perdre au public la perception du clown en train de jouer devant eux, et laisserait apparaître un acteur en prise avec son questionnement sur la justesse de son jeu. Le jeu porte ici sur l'absence de doute, de censure, sur la capacité à restituer ce que le clown vit dans l'instant, à savoir sur la capacité à donner du jeu à son clown. Tout ce que fait le clown est bon, tout ce qui est doute pour l'acteur devient certitude pour le clown. Contrairement à la poupée du ventriloque ou au mime, ou encore au bouffon, qui utilisent une enveloppe extérieure ou des traits d'un personnage, le clown existe comme étant soi-même rendu expressif dans certains états de corps. Comme le dit une participante à un stage: «Le clown c'est nous, sans le contrôle social que l'on exerce sur nous-même. » Si le maquillage n'est pas indispensable pour faire exister le clown, il aide néanmoins l'acteur - en tant que masque -à mettre de la distance entre son «moi social» et ce qu'il montre de lui dans son clown. À ce titre, le masque neutre - utilisé comme exercice d'apprentissage - peut être un outil puissant pour révéler un jeu de corps pour un clown: le visage masqué derrière un visage blanc sans expression, permet de rendre visible un corps qui peut se prêter à un jeu de clown. Lacteur, protégé par son masque, pense moins à des gestes convenus, il peut libérer une expressivité singulière. Du reste, on dit que le nez de clown, devenu optionnel dans le clown contemporain, représente le plus petit masque du monde. 


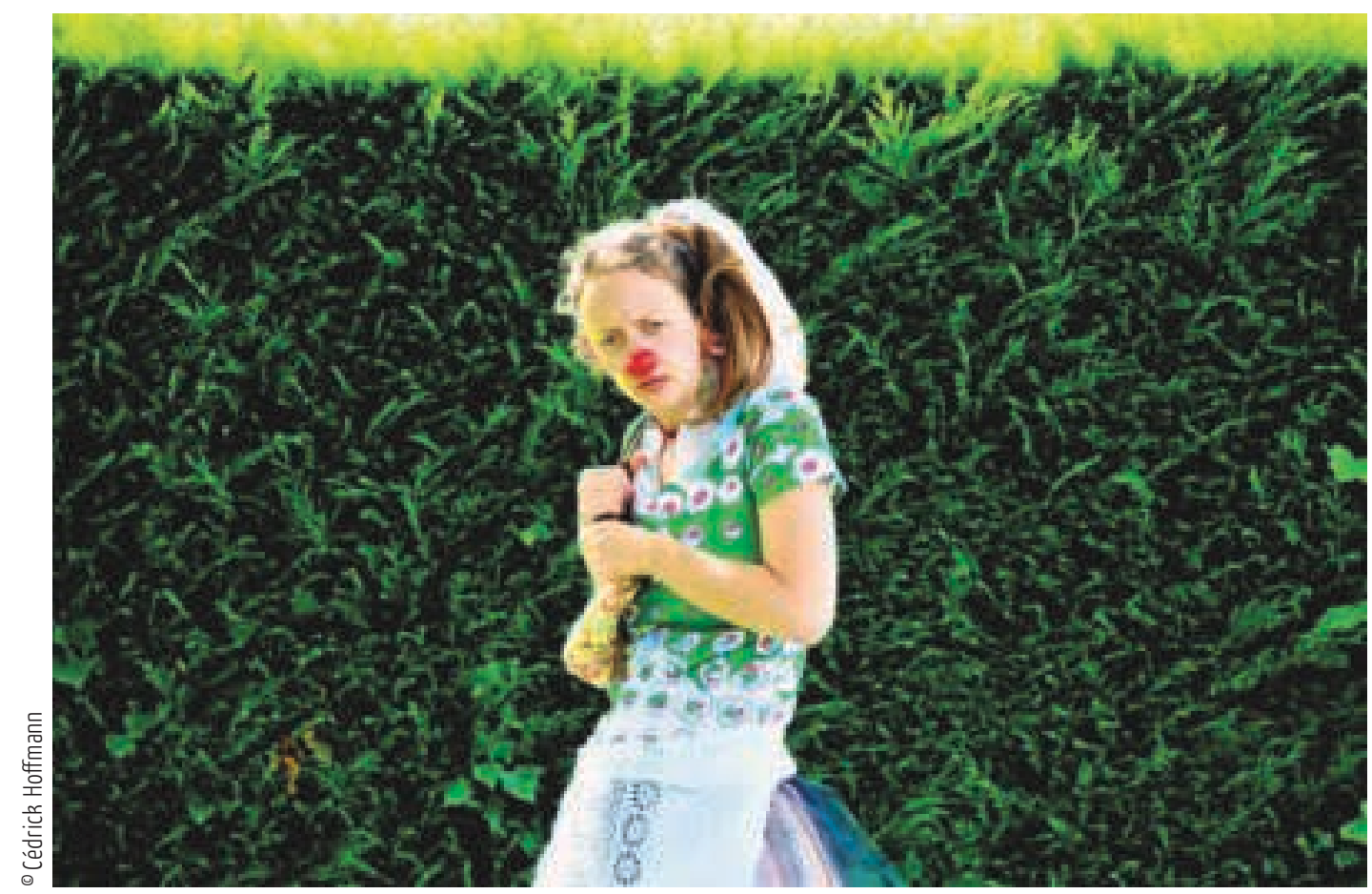

\section{Le corps outil du clown}

Pour parvenir à cet état d'acceptation, de non-doute, de non-censure, une seconde technique du corps porte sur l'isolement de sensations internes. Contrairement à la technique précédente du «saut dans le vide» qui procède par saturation, celle-ci permet à chaque apprenti clown de sérier des éléments corporels expressifs en un vocabulaire personnel efficace. Des techniques corporelles issues du théâtre sont parfois utilisées (Lecoq, Malmgren ${ }^{9}$ ). Elles utilisent pour guider le jeu du clown les sensations qu'éprouve l'acteur. Ainsi, les exercices d'échauffement reprennent parfois des jeux d'enfance: 1, 2, 3 soleil, chat perché, etc. Danser, sauter, tomber, tourner, rouler, rester sur place, sont autant d'actions simples qui permettent de repérer et réinvestir corporellement les mouvements, les postures, les états, les gestes qui procurent à l'acteur une prise, non pas par rapport à ce qu'ils pourraient donner sur scène selon des critères esthétiques formels, mais pour les états physiques qu'ils procurent et qu'il pourra regénérer: sensation de légèreté, de poids, d'excitation, de calme, de fluidité, de tension, de vivacité, jeu en collectif ou jeu en solitaire, etc. De là, il peut développer un ensemble de postures qu'il saura mobiliser plus facilement en jeu: un état noué ou libre, rigide ou fluide, de bouillonnement ou de calme intérieur, des sensations de légèreté ou de lourdeur, une attention focalisée ou non, des mouvements rendus vifs ou soutenus par le type d'attention que l'acteur leur porte, etc. Ensuite vient un travail plus spécifique sur le corps: isoler des parties du corps (la tête, le cou, le bassin) pour contrôler des mouvements simultanés ou distincts, les dissocier ou créer des tensions dans certaines parties, pour construire une silhouette, une démarche, une attitude. L'acteur peut alors rappeler ce vocabulaire physique qu'il aura appris. À l'instar de la danse ou du théâtre, ce sont des états physiques qui sont

\section{Compagnie SisMa}

La compagnie sisMa se propose d'explorer la pratique du clown sous hypnose, dans le cadre de stages encadrés par une clown artiste et un praticien en hypnose eriksonienne. L'hypnose est utilisée pour pouvoir «jouer, être-là, imaginer, bouger, chanter, danser, et se décaler de ses habitudes et automatismes» (Clémentine Jolivet, [ie SisMa). 
travaillés, qui peuvent être basés sur la recherche d'une mémoire sensorielle comme dans la voie proposée par Stanislavski. Les exercices physiques servent à trouver des sensations intérieures qui seront ensuite rendues visibles par des postures, des mouvements, des mimiques. Si l'on peut rapprocher ici le travail du clown de celui du danseur qui se fabrique un corps comme un outil au service de l'expression artistique, qualifier le corps du clown comme étant son outil semble réducteur. Ses états physiques ne sont pas au service d'une performance, ils sont au service du plaisir du clown, donc de ce qui peut être une résultante du travail de son corps-outil, sans pouvoir garantir que ce corps éprouvera le plaisir recherché. Ce plaisir s'éprouve sur scène, il est réel, le clown ne le contrôle pas; le public perçoit en général la différence avec une performance qui simule le plaisir instantanément - mais le clown peut jouer à simuler le plaisir, et y éprouver un plaisir réel, ajoutant un niveau de lecture possible à une scène. Apprendre à rendre son corps expressif, passe par l'expérimentation intime, avec ses propres limites et doutes. Dans ce cas, on peut parler du corps-outil du clown, au sens où il y a bien apprentissage et maîtrise corporelle dans le jeu, à condition toutefois de ne pas évacuer cette dimension sensible et vécue en situation qui lui échappe. Il y a bien une écriture de spectacle dans le clown, comme dans le théâtre, mais cette écriture ne concerne pas uniquement des scènes à jouer. Elle porte également sur des états de corps, vécus sur l'instant, à remobiliser à partir d'une mémoire du corps, parce que déjà traversés, pour en faire une matière expressive. L'apprentissage de la spontanéité du clown passe également par ce travail de mise en forme et d'écriture du faire-rire.

\section{Le faire-rire du clown: un jeu avec ses limites}

Le faire-rire du clown contemporain, distinct des genres comiques ou burlesques, renvoie peut-être à une modalité particulière du rire que Jean Duvignaud identifie comme à la fois destructeur et créateur, au moyen de la mise à distance par rapport à l'institué qu'il produit et à cause de la négativité qu'il révèle en plongeant dans les «profondeurs indéchiffrables de l'humain» (Duvignaud 1999: 12), orientant ainsi le questionnement vers une anthropologie politique du rire. L'analyse du savoir faire rire esquissée dans cet article constitue un versant symétrique à une telle analyse des usages sociaux du rire.

Le clown contemporain s'inscrit dans toute une tradition de trickster, de fou du roi, de bouffon, figures qui incarnent la transgression et la rupture. Dans son état spontané, il joue avec les failles de l'acteur et crée une rupture qui ouvre sur une autre expression. Cette autre expression est ce que Michel Dallaire résume par la démesure du clown. C'est par les faiblesses et les échecs de l'acteur que le jeu démesuré du clown pourra s'affirmer. Le clown pointe ses et nos limites individuelles par sa dérision, et nous l'aimons - ou le détestons ${ }^{10}$ - parce que nous nous y retrouvons. Il parle des limites de notre humanité et décale notre regard ${ }^{11}$. C'est pourquoi il peut mener à la subversion, comme l'illustrent les clowns artivistes (Lemoine $\&$ Ouardi 2010) ${ }^{12}$, ou à de la pure poésie (Cervantès \& Germain 2009).

Comment fait-il rire alors? Autant le dire clairement: ce n'est pas en essayant d'être drôle que le clown sera efficace. C'est d'ailleurs une injonction commune pour les « clowns 


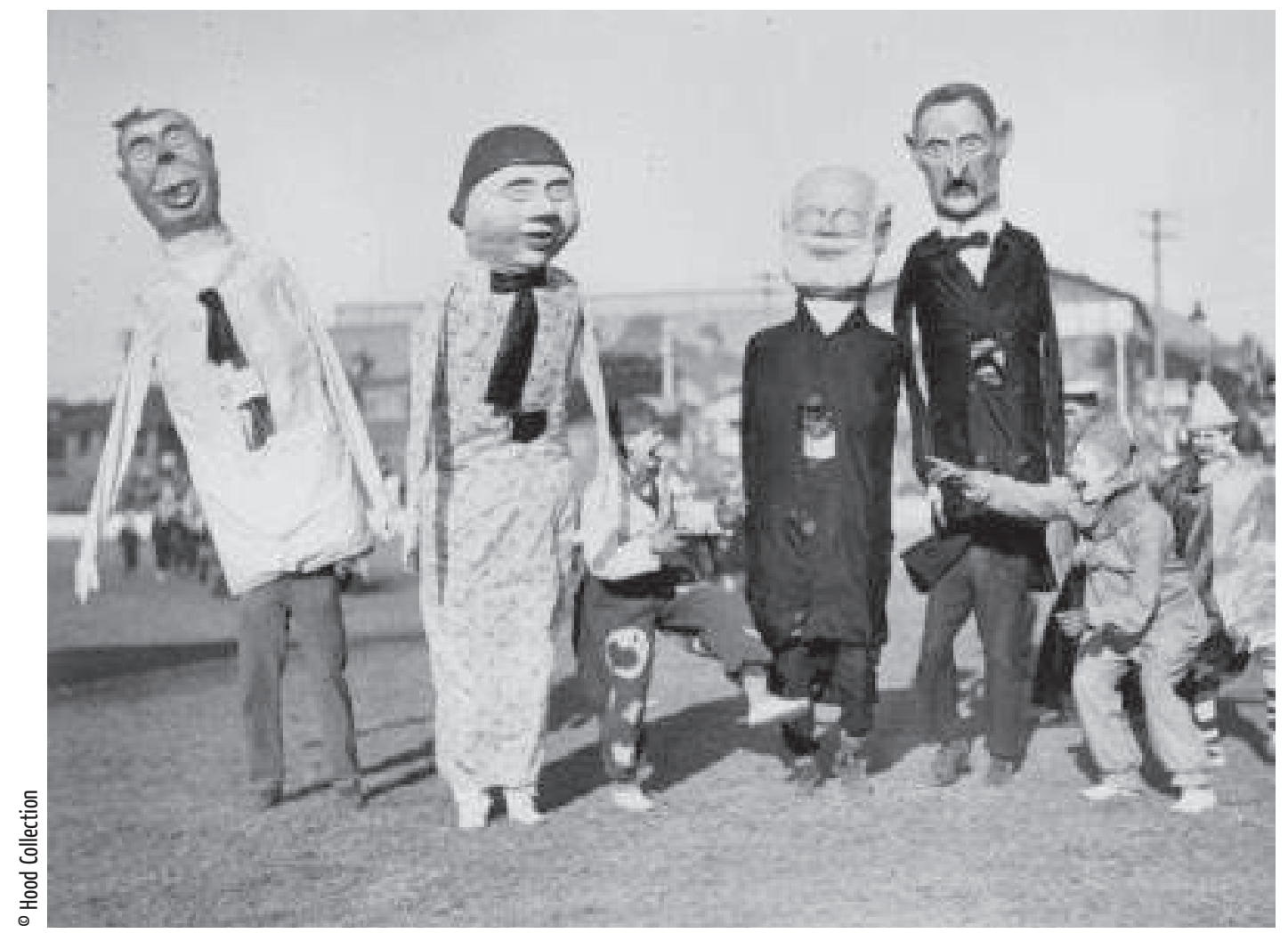

de stage $»^{13}$ : ne surtout pas faire le clown! S'il peut être tentant de chercher à exercer un pouvoir sur autrui à travers la capacité à faire rire, ce pouvoir ne peut s'exercer alors que dans un registre confiné, celui du rôle socialement attendu du clown de faire rire sur commande, qui étrangement ne correspond pas à la pratique effective du clown. L'efficacité, si elle existe, en tant que technique du corps, réside non pas dans la capacité à faire rire, mais plutôt dans la capacité de l'acteur à montrer ses propres limites. Le «mauvais clown » est celui qui n'intègre pas ce savoir faire, parce qu'il manque d'authenticité - il n’a pas de vécu intérieur et plaque un cliché de clown-, ou parce qu'il est un «faux» clown opportuniste - il porte un nez de façon stéréotypique mais sans exigence d'un travail -, ou qu'il est «raté»-vu comme un imposteur, se contentant de raconter des blagues sans avoir de jeu de clown (Cézard 2014: 157-164) ${ }^{14}$.

Lacteur doit trouver une efficacité dans son jeu, car il est attendu à l'endroit du rire, mais pas le clown. C'est là que les techniques du corps évoquées précédemment prennent toute leur dimension. Il importe de ne pas demander à «son» clown de faire le pitre, de faire rire, ce qui correspond pourtant à la figure populaire du clown. L'efficacité du travail de l'acteur se mesure à la réaction du public. Il attend de lui d'être drôle ou touchant. Être drôle: autre mot symétrique. Les clowns sont-ils drôles ou est-ce ce qu'ils font qui est drôle? Nous comprenons à présent mieux cette question. Le clown lui n'est pas dans la recherche d'efficacité, car le public n'y verrait que performance d'acteur. Il est dans la recherche de plaisir dans l'instant présent, sur scène, devant nous et avec nous, c'est pour cela qu'il est touchant. Plaisir et performance fonctionnent à l'inverse pour le clown et pour l'acteur: l'acteur ne joue pas le plaisir mais cherche l'efficace tandis

Clowns avec des gens portant de grandes figures de carnaval en papier-mâché, c. 1930 
que le clown cherche le plaisir et ignore l'efficace. Autrement dit, le moteur du faire-rire pour le clown n'est pas dans les formes culturelles attendues. Je propose l'hypothèse que c'est le corps du clown qui prête à rire, dans sa présence jubilatoire décalée, dans son rapport aux objets et aux autres, et non pas ce qu'il fait qui pourrait renvoyer à des formes attendues du faire-rire ${ }^{15}$.

Mais alors, que fait le clown sur scène? Il ne fait rien, il se contente d'être, ou plutôt tout ce qu'il fait n'est que prétexte pour être. Ce travail de l'acteur qui consiste à ne rien faire est clairement indiqué dans le travail de création du clown, comme le relève Delphine Cézard : «ne rien faire, être nul et tout rater» (2014: 115). La préparation du clown est l'apprentissage d'un savoir ne rien faire. Il consiste en un travail sur le silence, sur le temps suspendu, sur la sensation, sur les états de corps, entendez, le clown apprend à travailler des expressions d'un corps situé dans l'espace et le temps. Le travail permet de mieux montrer et faire entendre le dessous des mots : là encore le clown se manifeste dans sa présence corporelle, au-delà de la performance d'acteur, ou de danseur ${ }^{16}$. Le corps du clown dit au public: regardez ce que ça me fait, ce que je vis. La question est moins celle de l'esthétique - bien qu'il existe une esthétique du clown, comme il existe une esthétique de tout corps sur scène - que celle d'un corps ouvert et réceptif à des sensations face à un public. Il faut ajouter la jubilation à la description de cette présence corporelle du clown. La forme clown (le processus) renvoie à une certaine présence scénique, qui généralement engendre le rire, et qui n'est pas limitée à une forme de spectacle appelé «le clown» (le résultat de l'action). Le clown n'est pas un rôle que l'on joue mais un état, il est donc possible de l'appliquer à différents arts: il peut y avoir du clown dans bien d'autres formes contemporaines, entre théâtre, danse, musique, arts du spectacle.

\section{Le prétexte à l'expression de létat de clown}

Dans ce jeu scénique, l'apprenti clown risque d'en faire trop, de vouloir être original à tout prix, et d'être déconnecté de ses sensations propres. À l'inverse, la séquence jouée par Jos Houben est très banale: il s'appuie, glisse, tombe, se relève, marche. Il maîtrise parfaitement tous ses gestes, qui s'enchaînent de manière très précise. Mais il n'importe pas seulement de maîtriser des gestes, il faut également les incarner pour que le clown les vive. Ce comique millimétré - comique de geste ou de situation - nous fait rire, parce qu'il y a un décalage entre ce que fait le corps et l'intention. Bergson a proposé la formule devenue célèbre du rire comme étant provoqué par «du mécanique plaqué sur du vivant ». Il indique par là que l'on peut imiter de nos gestes: «que ce qu'ils ont de mécaniquement uniforme et, par là même, d'étranger à notre personnalité vivante. Si cette imitation peut faire rire, c'est parce qu'elle permet de dégager la part d'automatisme qui s'est introduite dans une personne» (2013: 17). Lacteur sait ce que son corps exprime, puisqu'il l'a abondamment travaillé, souvent face à un miroir et avec un regard extérieur. Mais ce qui fait rire est la réaction du clown, sa surprise, sa colère, son étonnement d'être aussi stupide. À côté de la mécanique huilée du corps sur scène se trouve une autre performance liée à la présence et l'écoute. Je dirais que ce n'est pas ce corps mécanique qui fait le clown, même s'il y participe, mais l'expression des sensations qu'il vit. Les chutes et accidents, forcément caricaturaux, sont autant de prétextes à l'expression de l'être clown vivant derrière ce numéro bien rodé. C'est l'état de corps du clown 
qui est drôle, état qu'il partage avec le public, en décalage avec une intention annoncée comme prétexte. Afin que ce corps de clown soit expressif, l'acteur a dû revenir à sa mémoire du corps, à des gestes expressifs et se débarrasser de tous les gestes parasites. On touche ici au «corps poétique» de Lecoq - maître de l'art du mouvement, fondateur du Laboratoire d'étude du mouvement -, qui envisageait le corps comme une écritureà part entière, avec ses mouvements, sa dynamique, ses équilibres et déséquilibres. Le vocabulaire physique de l'acteur, basé sur sa mémoire du corps et de ses sensations, lui permet de jouer ce qu'il y a d'essentiel, peut-être d'universel, dans une séquence. Les gestes, les mouvements et expression des corps, dans leur «naturalité» sont conscientisés et amplifiés, pour devenir expressifs; car «le corps se souvient de choses insoupçonnées que les enseignants essaient de réveiller» (Huthwohl 2012: 42). Nous retrouvons ici la dimension paradoxale déjà évoquée, où les gestes doivent être maîtrisés pour que puisse s'exprimer une spontanéité. Les gestes s'incarnent dans la présence du clown qui vient partager avec le public ce que c'est que de vivre dans son corps, ce que l'acteur joue. Le «regard public» du clown est important ici, c'est-à-dire sa complicité avec le public, pour attester ce qu'il est en train de vivre. Le clown décroche de l'action pour la vivre avec le public ou le partenaire.

Le clown ne fait pas de "comédie», il vit ses failles (frustration, vexation devant le ridicule qu'il montre, colère, impatience, orgueil, inattention, etc.), les amplifie éventuellement, et les partage avec le public. C'est ce qui offre une prise au clown et lui donne sa jubilation, comme l'indique le témoignage d'une clown en formation: «On ne cherche pas à forcément à faire rire, il suffit de s'amuser en côtoyant notre "erreur de casting" face aux autres! On se laisse aller à beaucoup d'improvisations quand on se mesure à ses nombreuses limites [...]. Ce qui est beau c'est de voir des "montées d'état" spectaculaires quand on se retrouve en décalage complet, pour aller dans des univers de plus en plus fous! ${ }^{17} »$ Le jeu du clown part de ce que son corps lui indique, parfois dans des micro-expressions, tels la panique, l'étonnement, la surprise, l'embarras, la jubilation, face à une situation. Le jeu d'acteur sert à construire cette situation jusqu'au point où elle échappe au jeu prévu, pour donner un espace d'expression au clown. Lacteur joue et le clown improvise.

\section{Une piste d’analyse de «l'état de clown»}

En conclusion, on peut dire que la spécificité du clown, par rapport aux autres arts de la scène, porte sur le jeu entre une performance d'acteur, une capacité à montrer et vivre ses émotions, ses sensations et à partager un état avec le public. Cet état est souvent ridicule, et montre par là une personnalité vivante comme le dit Bergson (2013). Si l'identité du clown contemporain suit un processus d'artification (Cézard 2014) en vue de le positionner dans le champ des arts, et se distinguer du registre traditionnel du clown, mon propos a porté ici sur une autre question, à savoir celle des techniques du corps qui permettent ou non de définir le clown, à partir de ses pratiques. À côté de la construction d'une identité de clown, je pose la question de la spécificité des pratiques du clown. Même si les comédiens ou les humoristes contemporains utilisent l'univers du clown - dans l'autodérision ou l'interpellation directe du public - il ne s'agit pas pour eux d'être ridicule «pour de bon ». Le clown ne joue pas à être ridicule, il accepte entièrement de l'être réellement comme condition même de son jeu, et l'offre au public à travers son regard. 
Où situer alors le clown: face aux arts du cirque, qui le renvoient à son jeu corporel, ou face aux arts de la scène et du théâtre, ou encore face au mime et au burlesque, qui le renvoient à son rapport au rire, face à l'écriture poétique enfin, que nous avons aperçu avec le clown Arletti (Cervantès \& Germain) ? Si le clown se caractérise davantage par ce qu'il est, que par ce qu'il fait, il constitue sans doute une piste intéressante pour préciser la notion de technique du corps. François Sigaut (2010) a proposé une relecture de la définition de Mauss, afin de mieux rendre compte de ce que peut signifier un «acte traditionnel efficace», en procédant par élimination. En soustrayant successivement chacun des termes de la définition (l'action, la tradition, l'effet), il met à jour et permet de regrouper un grand nombre de situations, qu'il articule à quatre registres d'activité: faire, apprendre, jouer, montrer. Dès lors, le clown se situe dans un ensemble d'activités qui relèvent du théâtre, du mime, des feintes. Il s'agit d'une action traditionnelle (culturelle) qui n'a pas d'effet, qui porte seulement sur les fauxsemblants et les doubles. Ce type d'action joue un rôle anthropologique, au sens où «toutes les sociétés inventent des jeux, des exercices ou des spectacles dans lesquels l'action traditionnelle efficace est analysée par la suppression, réelle ou fictive, de l'une de ses composantes» (Sigaut 2010 : 365). Le clown se distingue des autres jeux sur le double, dans le sens où le double de l'acteur qu'est le clown n'est pas simulé, il existe «pour de bon». Peut-être pourrait-on utiliser la description de Sigaut pour définir, dans un registre d'activité donné, en l'occurrence ici celui du «montrer», quel mode de présence corporelle est impliqué. Une telle analyse dépasse le cadre de cet article, mais constitue une piste pour préciser davantage ce que sont les «actions sans effet». Différencier le type de présence corporelle du clown de celle d'autres pratiques de la scène, permet de mieux situer le clown par rapport à ces autres pratiques. Le clown est dans un état de disponibilité corporelle, en plus d'être dans un faire - comme au cirque -, ou dans un faire semblant - dans du mime ou du théâtre, dans des registres qui relèvent du jeu d'acteur ou du comique et du prétexte de jeu.

Cette disponibilité corporelle permet de rendre compte de l'état de «lâcher prise» du clown, qui peut se traduire par une expressivité corporelle spécifique, vécue et partagée avec un public. Même s'il est question d'une maîtrise du corps dans cette expressivité, l'improvisation avec le public, le jeu sensible dans le plaisir et le partage du ridicule vécu, le regard adressé au public en lui offrant un état de corps vécu, contribuent à rendre vivant le clown, et le différencient d'autres formes de présence scénique, et d'actions sans effet, qui dépasse les jeux de faux-semblants. Le clown existe davantage par cette présence que par ses actes. L’action efficace, du corps maîtrisé, renvoie à une forme de présence corporelle sans attente et sans prétention. La notion de prise que j'ai mobilisée permet d'expliciter cette double dimension de l'action et de la non-action: l'acteur travaille corporellement pour trouver des manières de libérer une expressivité «spontanée» du corps. Il agit pour pouvoir laisser être. D’autres notions pourraient être utilisées pour décrire l'activité du clown - comme celles de représentation, de valeur esthétique ou culturelle, d'identité - mais elles ne permettent pas de signifier cette manière de se rendre sensible à son corps et aux situations, tout en contrôlant les effets partagés ou non de ses états de corps. On retrouve l'approche constructiviste et réflexive d'une analyse pragmatique de l'action. Si on pousse un peu le parallèle avec ce que Bessy et Chateauraynaud (2010) décrivaient à propos des investissements de forme auxquels s'emploient les experts pour pouvoir laisser parler la matière (laisser parler l'authenticité d'un tableau par exemple), ici la «matière » est le corps de l'acteur. 
Il y a un savoir être clown qui repose sur une préparation du corps et un savoir faire de cette présence. Le clown sait dépasser le paradoxe d'un corps maîtrisé dans un jeu calibré pour qu'une expression spontanée de ce corps puisse apparaître. Une sorte de paradoxe du comédien appliqué au corps. Le cas de Jos Houben entre autres, indique un enchaînement de gestes très précis. Il s'illustre dans sa fonction de pédagogue qu'il est également, il enseigne à l'école Jacques Lecoq -, cependant son clown apparaît dans le ridicule de son personnage de clown-professeur, qu'il a plaisir à nous montrer. Tandis que son discours et ses explications sont impeccables, son clown s'immisce dans son jeu corporel qui déséquilibre le propos sérieux et le décale. Le propos devient le prétexte pour faire rire. Un autre clown (personnage) ferait un autre clown (état). Le corps ainsi travaillé spécifiquement n'est-il alors pas précisément le lieu d'apparition du plaisir de «l'être clown»? La catégorie heuristique du clown résiderait dans son jeu corporel, dans une parole donnée à un corps devenu expressif, paradoxalement grâce au travail de maîtrise dont il a fait l'objet. Ce dernier propos semble objectaliser le corps du clown. Or tout ce qui précède montre que le corps du clown n'est en rien un objet manipulé par un acteur.

Enfin, je propose l'idée d'un corps politique du clown: la présence scénique du clown montre un «être-corps» qui dit les limites du jeu d'acteur, du jeu social, pour indiquer une nature humaine plus profonde dont il témoigne par sa jubilation... Qu'est-ce qui joue alors dans le clown? Il est question d'un corps rendu vivant par un travail, au sens où, en jouant de ses limites, il incarne une libération des convenances corporelles, scéniques et sociales, et témoigne, dans sa jubilation, de ce que peut exprimer un corps, car ce corps est en même temps maîtrisé. Ce sont peutêtre là quelques premières pistes de compréhension de ce que des apprentis clowns cherchent dans cette pratique.

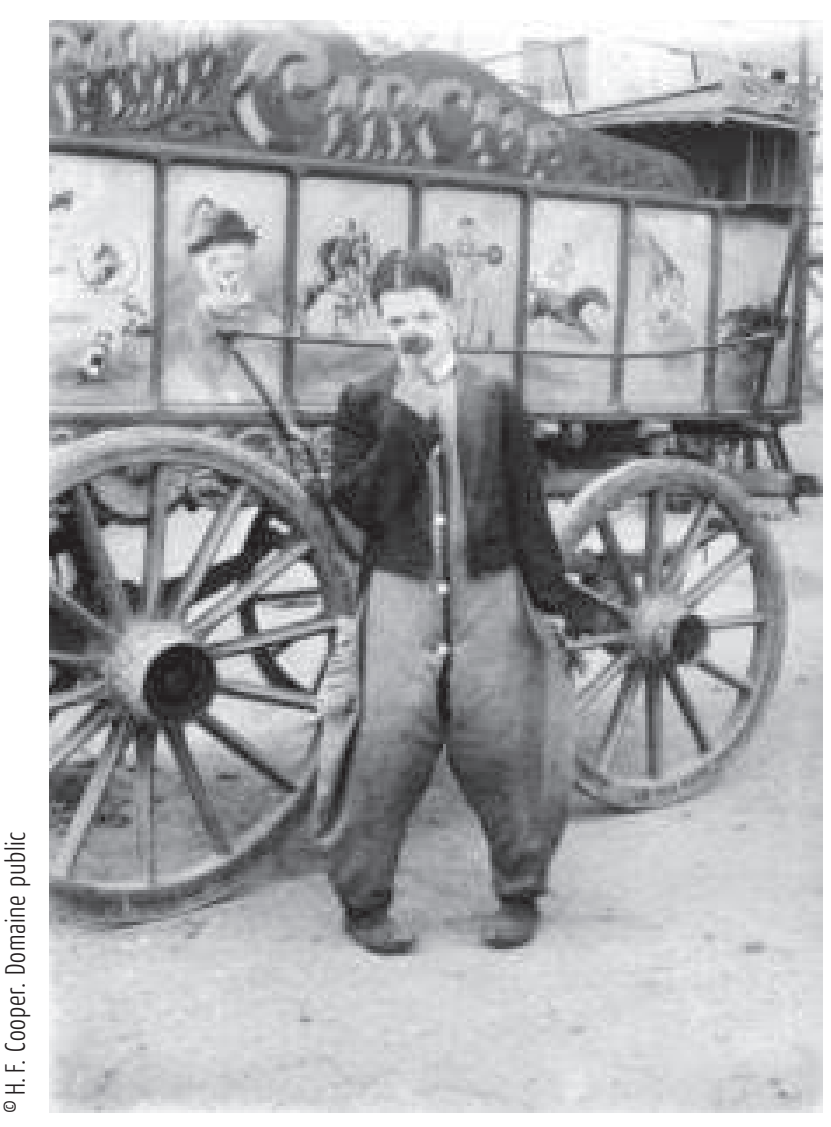

The Grand Circus, c. 1910

Public Record Office of Northern Ireland 


\section{NOTES}

Photo d'ouverture: Jos Houben, L'art du rire. Extrait d'une vidéo du spectacle de Jos Houben L'art du rire ${ }^{18}$, qui fait une leçon en clown sur le clown. Il explique des «trucs» qui marchent, les exécute (ici le «gag» classique du serveur qui accumule les maladresses en voulant servir un client imaginaire: trébucher sur la chaise, y coincer sa main, renverser la carafe d'eau, laisser tomber une serviette, à plusieurs reprises), et ça fait rire. Le fait de dévoiler l'astuce de l'acteur n'enlève pas le plaisir, ni le caractère éculé du «gag», qui ne sert ici que de prétexte. Ce qui fait rire est la réaction du clown qui s'est, une fois de plus, fait prendre à son jeu... Dans le comique de répétition, en clown, ce n'est pas uniquement la technique narrative de répétition qui amène le rire, mais également l'état produit sur le personnage de l'action répétée.

1. Compagnie Les Passe-Murailles, Michel Rousseau, entretien janvier 2014.

2. Michel Dallaire, Lexique, Le Hangar des Mines, SaintSébastien-d'Aigrefeuille, 2013.

3. Michel Dallaire, id.

4. Entretien, juillet 2015.

5. Les techniques du Body Mind Centering (BMC) sont notamment utilisées par les danseurs, comme moyen pour aiguiser la perception de leur propre corps (Bainbridge Cohen 2002). N'ayant pas suivi de formation ou de stage en BMC je ne préciserai pas davantage le type de travail mis en place.

6. Entretien, Le Hangar des Mines, avril 2012.

7. Voir la chaîne Youtube «Quai TV Angers », www. youtube.com/watch?v=dzJF5caATPs.

8. Compagnie Self Retorik, Romain Yvos, entretien mai 2014.

9. Yat Malmgren (1916-2002), fondateur au Drama Center de Londres d'une méthode d'expression de l'état intérieur d'un personnage par le mouvement, qui est une synthèse de la théorie de Rudolph Laban de l'expression du mouvement, des quatre types de personnalité de C. G. Jung et les principes clés de l'action établis par Constantin Stanislavski.

10. Le clown peut éveiller de l'enthousiasme, mais également du rejet, de la peur, voire de la condescendance. Il illustre par là le fait que l'artiste laisse entrevoir des parts de nous que nous acceptons plus ou moins facilement.
11. Se pose ici la question de l'universalité du clown. Des formes de clown se retrouvent-elles dans toutes les cultures? Quelles sont ses modes d'expression, le faire rire est-il culturel? Tout un champ de questionnements se dessine qui pose à la fois la question des formes culturelles changeantes de l'expression clownesque et de la réception universellement partagée de ce qui déclenche le rire.

12. Les Clandestine Insurgent Rebel Clown Army, et les Yes Men, pour ne citer que les plus connus. Le terme artivisme est un néologisme, contraction d'activiste et d'artiste.

13. Spectacle d'Alain Gautré, Le gai savoir du clown, conférence drôlatique.

14. Ces distinctions participent du processus d'artification du clown contemporain selon Cézard (2014).

15. Même s'il peut également parodier ces formes conventionnelles. À ce propos, un formateur à l'école de clown du Hangar des Mines dit: «c'est très difficile pour un clown de faire ce que j'appelle le "clou-clown" des animations de supermarché, il est très fort celui qui y arrive! » (entretien avril 2012).

16. Un lien peut être envisagé avec la danse, le butô en particulier, où le ridicule et le grotesque sont également revendiqués.

17. Compagnie Self Retorik, entretien mai 2014, stage.

18. Voir la chaîne Youtube du «Théâtre de Vénissieux - L'art du rire de Jos Houben», www.youtube.com/ watch?v=blOWjTDDh6k.

\section{POUR CITER CET ARTICLE}

Hert, P. 2017 «Apprendre à faire le clown» in G. Bartholeyns \& F. Joulian, Le corps instrument, Techniques \&Culture 62: 30-47. 


\section{RÉFÉRENCES}

Bainbridge Cohen, B. 2002 Sentir, ressentir, agir. L'anatomie expérimentale du Body-Mind Centering. Bruxelles: Contredanse.

Bergson, H. 2013 [1900] Le rire. Paris: Flammarion.

Bessy, Ch. \& F. Chateauraynaud 1995 Experts et faussaires. Pour une sociologie de la perception. Paris: Métailié. Bessy, Ch. \& F. Chateauraynaud 2010 «Retour sur "Le savoir-prendre" », Techniques\&Culture 54-55 (2): 686-688.

Cézard, D. 2014 Les «nouveaux» clowns. Approche sociologique de l'identité, de la profession et de l'art du clown aujourd'hui. Paris: L'Harmattan.

Duvignaud, J. 1999 Rire et après? Essai sur le comique. Paris: Desclée de Brouwer.

Germain, C. \& F. Cervantès 2009 Le clown Arletti: Vingt ans de ravissement. Paris: Magellan et C Cie.

Hennion, A. 2009 « Réflexivités. L'activité de l'amateur », Réseaux 153: 55-78.

Huthwohl, J. 2012 «École de création, école de la vie. Chez Jacques Lecoq. », Revue de la BNF 2012/1 (40): 40-43.

Lecoq, J. 1997 Le Corps poétique. Arles: Actes Sud.

Lemoine, S. \& S. Ouardi 2010 Artivisme: art, action politique et résistance culturelle. Paris: Éditions Alternatives. Sigaut, F. 2010 [2002] «La formule de Mauss », TechniquesECulture 54-55 (1): 357-367.

\section{RÉSUMÉ}

Apprendre à faire le clown. Nous proposons d'aborder l'apprentissage de la pratique du clown en l'analysant à partir de la place du corps. Cet apprentissage est abordé en tant que technique du corps et en tant que recherche de prises que le clown trouve pour exprimer son jeu. Il implique une expérimentation d'états de corps face à un public. L'apprentissage du clown, en plus d'un jeu d'acteur, intègre l'expression des sensations et des émotions qu'il vit réellement en situation. Nous posons donc la question: qu'est-ce qui joue dans le clown qui nous fait rire? Comment peut se définir l'être clown? En mettant en évidence que c'est dans le jeu avec ses propres failles - failles que nous partageons tous - que se définit l'usage du corps du clown, nous essayons d'y voir un principe structurant.

\section{ABSTRACT}

The art of doing the clown. The learning of practicing the clown is analyzed here from the place of the body. This learning is approached as a body technique and as a way to take reach of ways to express the clown's game. It involves to experiment body states in front of an audience. Learning the clown, in addition to acting, incorporates the expression of feelings and emotions that he lives in situation. We therefore ask: what plays in acting as a clown that makes us laugh ? How can be defined being a clown? Highlighting that it is the game with its own flaws -flaws that we all share - that may define the use of the body of the clown, we try to see here a structuring principle.

\section{MOTS CLÉS}

Techniques du corps, prise, états de corps, jeu scénique, présence, action, paradoxe du comédien

\section{KEYWORDS}

Body techniques, take reach, body states, acting, actor presence, paradox of the actor 